\title{
Superconducting Bolometer for Terahertz Detection
}

\author{
Mathias Kehrt, Christian Monte, Jörn Beyer, Jörg Hollandt \\ Physikalisch-Technische Bundesanstalt (PTB) \\ Abbestr 2-12, D-10587 Berlin, Germany
}

\section{Introduction}

Over decades the spectral range from $0.1 \mathrm{THz}$ to $3 \mathrm{THz}$ (the spectral range of far-infrared (FIR) or THz radiation) was covered by astronomic research. In the last years $\mathrm{THz}$ radiation became a growing field of interest in laboratory measurements, industry and homeland security. Supporting these efforts we are developing a composite bolometer for $4.2 \mathrm{~K}$ operation that addresses the special requirements of Fourier transform spectroscopy (FTS). The design meets the requirements of an existing calibration facility for spectral radiance in the range of infrared (IR) und FIR using FTS [1]. This facility operates under vacuum to avoid atmospheric absorption. To reduce thermal background radiation the beam line is cooled by liquid nitrogen $\left(\mathrm{LN}_{2}\right)$, also all relevant optical components are cooled. The aim is to provide a bolometer with high and uniform absorption over the whole spectral range from $0.1 \mathrm{THz}$ to $3 \mathrm{THz}$ and with a more linear response than commercial semiconducting bolometers which are currently used in FTS. Fourier transform spectroscopy requires a highly linear and fast detector to measure an interferogram. Especially linearity is critical in FTS because a non linear detector response leads to spectral artefacts, which can hardly be corrected. Both, the good linearity and the broad and uniform absorption, are the main requirements on the absorber as well as on the thermistor. Due to these requirements we decided to develop a composite bolometer with a transition edge sensor (TES) as thermistor for our applications. The composite design allows us to optimise the thermistor and the absorber separately.

The following section describes bolometers more generally and gives some details about the operation parameters specific for our application. The design of the thermistor and the absorber is described afterwards. At the end a summary is given.

\section{Bolometer}

Bolometers are thermal detectors based on a simple concept. Radiation heats an absorber with a heat capacity $C$. The absorber is thermally connected to a heat reservoir at temperature $T_{\text {res }}$ through a link with a thermal conductivity $G$. Measuring the temperature variation in the absorber allows to determine the incident radiant power [2]. Lower operating temperatures increase the sensitivity of the device. To detect lowest intensities the bolometer described here will be cooled by liquid helium and operated at a reservoir temperature $T_{\text {res }}=4.2 \mathrm{~K}$.

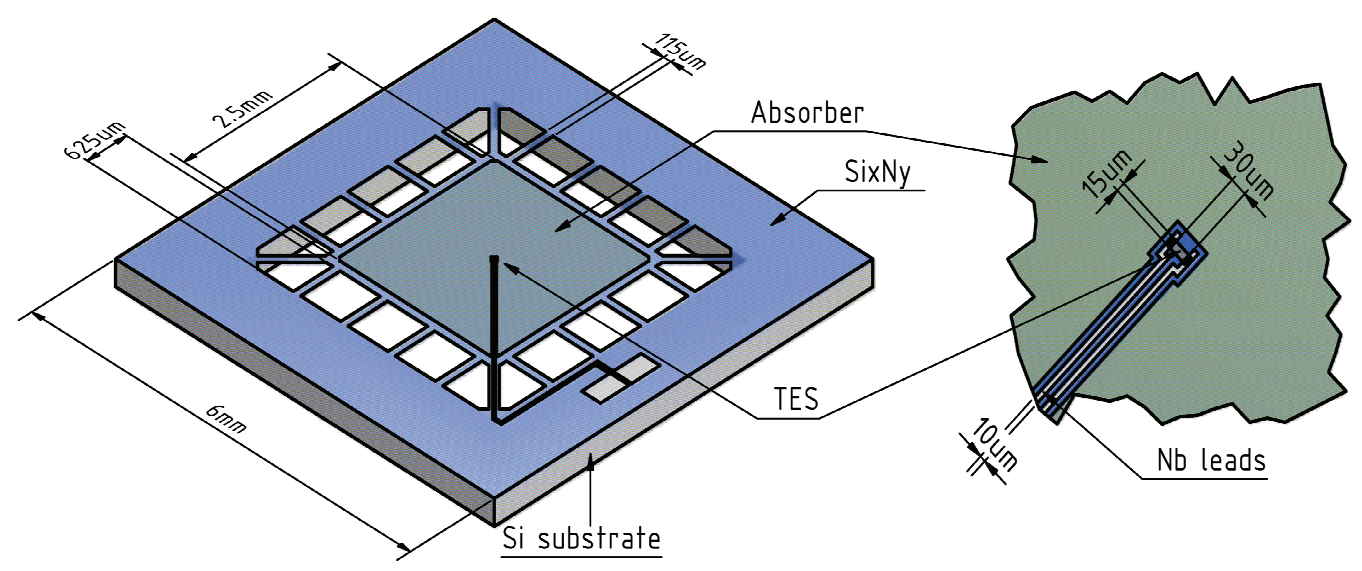

Figure 1: Design of the composite bolometer with transition edge sensor (TES) operated at $4.2 \mathrm{~K}$. The absorber will be realised by a single resistive micro-structured metal layer on a SiN membrane linked by bars to the heat reservoir with $T_{\text {res }}=4.2 \mathrm{~K}$. An Al/Nb bilayer with $\mathrm{Nb}$ wiring will be used as TES. 
The required operation parameters of the TES bolometer as part of the PTB FTS facility were estimated analytically. Assuming a blackbody at $300 \mathrm{~K}$ in front of the FTS and a $100 \mathrm{~cm}^{-1}$ longpass filter between the blackbody and the bolometer the interferogram shown in Figure 2 was calculated. This allows to estimate the expected dynamic range of alternating power incident on the detector of $P_{\mathrm{ac}}=11 \mu \mathrm{W}$. Additionally a constant offset $P_{\mathrm{dc}}=2.5 \mu \mathrm{W}$ was calculated assuming that the beam splitter inside the spectrometer emits temperature radiation at $300 \mathrm{~K}$ with an emissivity of 0.3 . The beam splitter illuminates the bolometer behind the $100 \mathrm{~cm}^{-1}$ longpass filter without interfering in the FTS. This results in an expected maximum radiant power of $13.5 \mu \mathrm{W}$ on the bolometer for a typical application of the FTS.

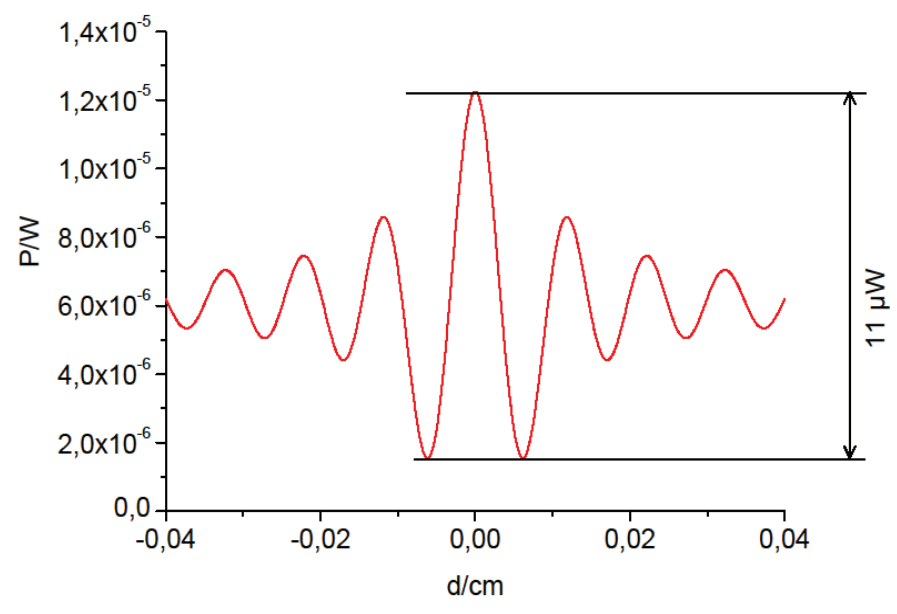

Figure 2: Expected dynamic range of radiant power at a detector inside of the Fourier transform spectrometer assuming a $300 \mathrm{~K}$ blackbody with a $100 \mathrm{~cm}^{-1}$ longpass filter in front of the detector.

\section{Superconducting Thermistor}

Low temperatures below $10 \mathrm{~K}$ are ideally suited to utilise superconducting devices. The resistive phase transition from superconducting to normal conducting state is characterised by a drastic increase of the electric resistance $R_{\text {TES }}$ within a very narrow temperature range. This transition edge can be used to implement a highly sensitive thermistor, called transition edge sensor (TES).

We are using an aluminium/niobium bilayer for the thermistor with a transition temperature $T_{\mathrm{c}}$ of about $8 \mathrm{~K}$ and pure niobium with $T_{\mathrm{c}}$ of about $9 \mathrm{~K}$ for the wiring connection. Aluminium is normal conducting above $1.2 \mathrm{~K}$. Due to the proximity effect the normal conducting aluminium reduces the transition temperature of niobium with respect to the wiring connection locally. This is shown in Figure 3 . The main curve shows the resistance of the whole assembly: TES and wiring as a function of temperature. Both transition edges are sufficiently separated. So the TES (bilayer) can be operated at its transition edge (left inset of Figure 3 ) while the wiring is still superconducting.

The TES will be voltage-biased with a constant voltage $V_{0} \approx l_{\text {bias }} R_{\text {bias }}$ as shown in Figure 4 . This results in a resistive heating of the TES given by an electrical power $P_{\text {bias }}=V_{0}^{2} / R_{\text {TES. }}$. Absorbed radiant power $P_{\text {abs }}$ leads to increasing temperature in the TES and so to increasing resistance $R_{\text {TES. }}$. An increasing resistance $R_{\text {TES }}$ leads to a decreasing heating power $P_{\text {bias. }}$. If the thermistor is biased in the steep part of the transition range the total power of the bolometer $P_{\text {total }}=P_{\text {bias }}+P_{\text {abs }}$ and therefore the temperature $T_{\text {TES }}$ will essentially

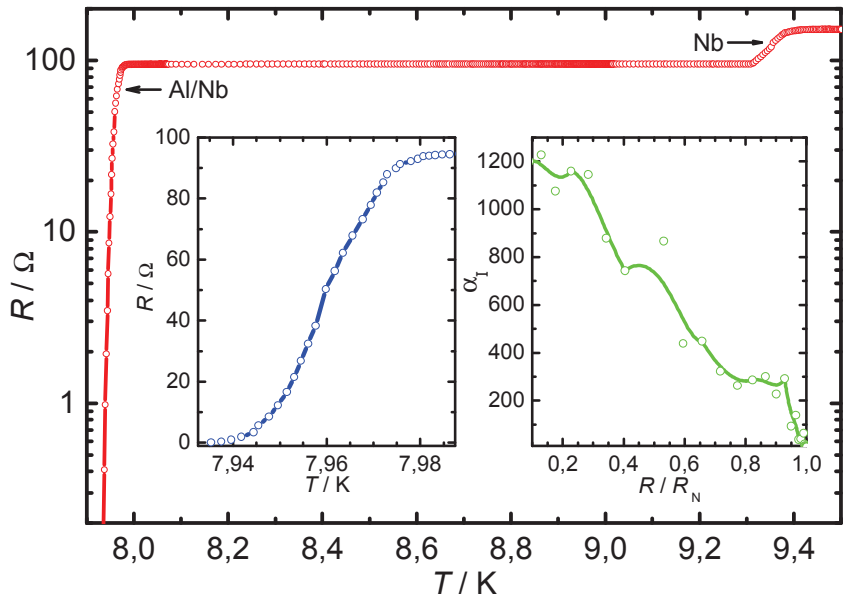

Figure 3: Measured resistance $R(T)$ of an Al/Nb TES. Main curve: Transistions of $\mathrm{Al} / \mathrm{Nb}$ bilayer and $\mathrm{Nb}$ wiring are marked. Left inset: Zoom in on the steep bilayer transition. Right inset: Normalised temperature coefficient $\alpha_{1}$ of the transition region.

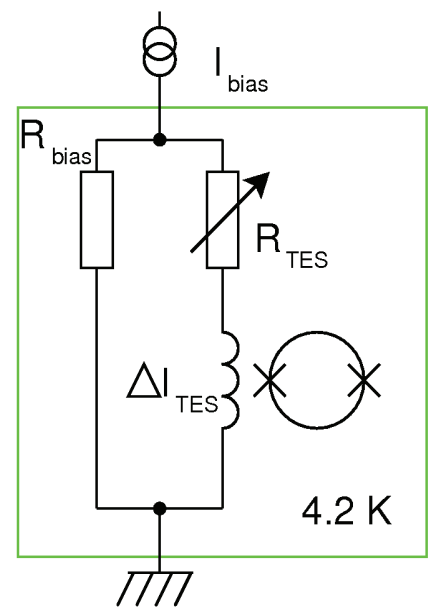

Figure 4: TES readout. The TES operates at constant voltage. The change of current through the TES is measured with a SQUID current sensor. $R_{\mathrm{bias}}$ " $R_{\mathrm{TES}}$. 
remain constant. This effect is known as negative electrothermal feedback (nETF) [3].

While the thermistor itself is just sensitive in the steep part of the transition (cf. Fig. 3, left inset), the nETF extends the dynamic range to $\delta P_{\text {abs, } \max } \leq G\left(T_{\text {TES }}-T_{\text {RES }}\right)$ with thermal conductivity $G$ of the link to the heat reservoir by reducing the Joule heating.

For the TES readout a 32-SQUID array current sensor with sufficiently low noise and high dynamic range was developed in-house.

To compare the sensitivity of various thermistors the normalised temperature coefficient $\alpha_{1}=(T / R) \cdot(\partial R / \partial T)$ is typically used. Conventional semiconducting thermistors typically have a temperature coefficient of $\alpha_{1} \approx-1 \ldots-10$. Superconducting thermistors have a significant higher temperature coefficient $\alpha_{1}$ of 10 up to 1000. The curve in the right inset of Figure 3 shows the measured normalised temperature coefficient of the TES. The region of interest is between $20 \%$ to $50 \%$ of the resistivity of the normal conducting state corresponding to a temperature coefficient $\alpha_{1} \approx 500$ to 1000 .

\section{Absorber}

Design parameters of the absorber are a high and uniform absorption in the range of $0.1 \mathrm{THz}$ to $3 \mathrm{THz}$. A first approach to realise a high and uniform absorption is to match the vacuum impedance with the sheet resistance of the absorber [4]. A resistive absorber layer with a sheet resistance of $188 \Omega$ would be desirable. Typical metals have a resistivity of $10 \Omega \mathrm{nm}$ to $1000 \Omega \mathrm{nm}$ which would lead to a thickness of a single-layer absorber of around $1 \mathrm{~nm}$. More practically, however, normal metal layers with a thickness of $10 \mathrm{~nm}$ to $100 \mathrm{~nm}$ can be fabricated reproducible and in good quality. In order to match the vacuum impedance, such metal layers can be micro-structured with characteristic lateral dimensions smaller than the shortest wavelength of interest. Optimised structures are expected to be efficient and broad-band $\mathrm{THz}$ absorbers.

First test structures were fabricated in-house with conventional photolithography. Shown in Figure 5 are quadratic grids with ring structures in the middle of the

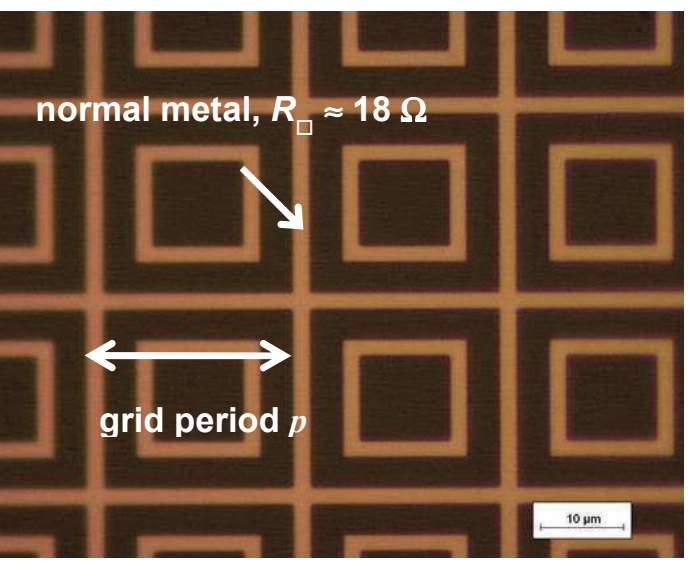

Figure 5: Absorber surface with microstructured metal grid and ring structure in the middle of the mesh.

mesh. The line width is chosen as $3 \mu \mathrm{m}$. Currently, a series of test structures is fabricated with different grid periods $p=20 \mu \mathrm{m}$ to $50 \mu \mathrm{m}$ for systematic investigation of their $\mathrm{THz}$ absorptance.

\section{Summary}

The PTB is developing a composite bolometer which is optimised for an existing calibration facility in the $\mathrm{THz}$ spectral range using Fourier transform spectroscopy. The aim is to cover the spectral range from $0.1 \mathrm{THz}$ to $3 \mathrm{THz}$ with respect to the special needs of FTS, namely good linear response over a broad dynamic range and a uniform absorption over a broad spectral range. Therefore absorber and thermistor are optimised separately.

The composite bolometer is designed to operate at $4.2 \mathrm{~K}$. To extend the dynamic range a superconducting thermistor is voltage biased in a loop with negative electrothermal feedback. SQUIDs will be used as a current sensor to realise the readout.

For a high and uniform absorption from $0.1 \mathrm{THz}$ to $3 \mathrm{THz}$ absorbers which are designed as a microstructured metal layer, are investigated.

[1] Christian Monte, Berndt Gutschwager, Svetlana Morozova, and Jörg Hollandt. Radiation Thermometry and Emissivity Measurements Under Vacuum at the PTB. International Journal of Thermophysics, 30, 203-219, (2009).

[2] P. L. Richards. Bolometers for infrared and millimeter waves. Journal of Applied Physics, 76, 1-24, (1994).

[3] Adrian T. Lee, Paul L. Richards, Sae Woo Nam, Blas Cabrera, and K. D. Irwin. A superconducting bolometer with strong electrothermal feedback. Applied Physics Letters, 69, 1801-1803, (1996).

[4] J. J. Bock, D. Chen, P. D. Mauskopf, and A. E. Lange. A novel bolometer for infrared and millimeterwave astrophysics, Space Science Reviews, 74, 229-235, (1995). 